\title{
On discreteness of commensurators
}

\author{
MAHAN MJ
}

\begin{abstract}
We begin by showing that commensurators of Zariski dense subgroups of isometry groups of symmetric spaces of noncompact type are discrete provided that the limit set on the Furstenberg boundary is not invariant under the action of a (virtual) simple factor. In particular for rank one or simple Lie groups, Zariski dense subgroups with nonempty domain of discontinuity have discrete commensurators. This generalizes a Theorem of Greenberg for Kleinian groups. We then prove that for all finitely generated, Zariski dense, infinite covolume discrete subgroups of $\operatorname{Isom}\left(\mathbb{H}^{3}\right)$, commensurators are discrete. Together these prove discreteness of commensurators for all known examples of finitely presented, Zariski dense, infinite covolume discrete subgroups of Isom $(X)$ for $X$ an irreducible symmetric space of noncompact type.
\end{abstract}

$57 \mathrm{M} 50$

\section{Introduction}

Motivated by Margulis' celebrated characterization of arithmeticity of irreducible lattices in semisimple Lie groups in terms of density of the commensurator [18], Shalom asked (see Leininger, Long and Reid [16]) for a description of commensurators of Zariski dense subgroups of semisimple Lie groups. We start with the observation that for Zariski dense subgroups of isometry groups of rank one symmetric spaces, commensurators are discrete provided that the domain of discontinuity is nonempty.

Proposition 1.1, Corollary 1.2 Suppose $\Gamma$ is a Zariski-dense subgroup of a semisimple Lie group $L=\operatorname{Isom}(X)$ for $X$ a rank one symmetric space of noncompact type. Then the commensurator $\operatorname{Comm}(\Gamma)$ of $\Gamma$ is discrete if the limit set $\Lambda_{\Gamma}$ is not all of $\partial X$.

When $X=\mathbb{H}^{3}$, the above is due to Greenberg [9; 10].

Using the theory of limit sets (in the Furstenberg boundary $Y=G / B$ ) of Zariskidense subgroups of semisimple Lie groups of higher rank developed by Benoist [2] we generalize Proposition 1.1 to arbitrary semisimple Lie groups.

Proposition 1.7 Suppose $\Gamma$ is a Zariski-dense subgroup of a semisimple Lie group $L=\operatorname{Isom}(X)$ for $X$ a symmetric space of noncompact type. Further suppose that the limit set $\Lambda_{\Gamma} \subset Y$ is not invariant under any nontrivial semisimple (virtual) factor subgroup $L_{0}$. Then the commensurator $\operatorname{Comm}(\Gamma)$ of $\Gamma$ is discrete in $L$. 
We then specialize to the case that $\Gamma$ is abstractly a (relative) hyperbolic group in the sense of Gromov and $X$ is of rank one.

Infinite covolume discrete subgroups of semisimple Lie groups have received little attention in the context of commensurators until recently. However, the theory of Kleinian groups deals primarily with infinite covolume discrete subgroups of $\mathrm{PSL}_{2}(\mathbb{C})$. Commensurators of some nonfree Kleinian groups have been investigated by Leininger, Long and Reid [16]. In this paper we reprove and extend their results to all finitely generated Kleinian groups.

Theorem 3.7 Let $G$ be a finitely generated, Zariski dense Kleinian group such that $\mathbb{H}^{3} / G$ has infinite volume; then the commensurator $\operatorname{Comm}(G)$ of $G$ is discrete in $\operatorname{PSL}_{2}(\mathbb{C})$.

The above statement was proven by Leininger, Long and Reid [16] when $G$ is nonfree and without parabolics. As in [16], Theorem 3.7 can be strengthened as follows.

Theorem 3.8 Let $G$ be a finitely generated Kleinian group such that $G$ is Zariski dense in $\operatorname{PSL}_{2}(\mathbb{C})$ and $\mathbb{H}^{3} / G$ has infinite volume. Then $[\operatorname{Comm}(G): G]<\infty$ unless $G$ is virtually a fiber subgroup, in which case $\operatorname{Comm}(G)$ is the fundamental group of a virtually fibered finite volume hyperbolic 3-manifold.

Together, Proposition 1.7 and Theorem 3.7 prove discreteness of commensurators for all known examples of finitely presented, Zariski dense, infinite covolume discrete subgroups of Isom $(X)$ for $X$ a symmetric space of noncompact type.

We also provide an example of a Zariski dense subgroup $G \subset \operatorname{Isom}\left(\mathbb{H}^{4}\right)$ such that $\operatorname{Comm}(G)$ is discrete, $[\operatorname{Comm}(G): G]=\infty$ but the limit set $\Lambda_{G} \neq \partial \mathbb{H}^{4}$. We also justify a statement of Gromov [12] by showing that negatively curved 4-manifolds cannot fiber over the circle.

\subsection{Zariski-dense subgroups of semisimple Lie groups}

Our first observation is that a version of Margulis' dichotomy holds for all Zariski dense subgroups. (Some version of this is probably well-known to experts in the theory of Lie groups and algebraic groups.)

Proposition 1.1 Suppose $\Gamma$ is a Zariski-dense subgroup of a semisimple Lie group $L=\operatorname{Isom}(X)$ for $X$ a rank one symmetric space of noncompact type. Then the commensurator $\operatorname{Comm}(\Gamma)$ of $\Gamma$ is either discrete or dense in $L$.

Proof Let $\overline{\operatorname{Comm}(\Gamma)}$ be the closure of $\operatorname{Comm}(\Gamma)$ in the Lie group $L$. If the connected component of the identity $L_{0}$ of $\overline{\operatorname{Comm}(\Gamma)}$ is trivial, then $\overline{\operatorname{Comm}(\Gamma)}$ and hence $\operatorname{Comm}(\Gamma)$ is totally disconnected and therefore discrete. 
Else, let $\mathfrak{l}_{0}$ denote the Lie algebra of $L_{0}$ and $\mathfrak{l}$ denote the Lie algebra of $L$. Since $L_{0}$ is the connected component of the identity of $\overline{\operatorname{Comm}(\Gamma)}, L_{0}$ is normal in $\overline{\operatorname{Comm}(\Gamma)}$. Then $\mathfrak{l}_{0}$ is an invariant subspace of $\mathfrak{l}$ under the adjoint action $\operatorname{Ad}_{\Gamma}$ of $\Gamma$. Hence $\mathfrak{l}_{0}$ is an invariant subspace of $\mathfrak{l}$ under the adjoint action of $\mathrm{ZC}(\Gamma)$, the Zariski closure of $\Gamma$. (This follows from the fact that $\operatorname{Ad}_{G}$ acts on the Grassmannian of $\operatorname{dim}\left(\mathfrak{l}_{0}\right)$ - planes in $\mathfrak{l}$ and $\operatorname{Ad}_{\Gamma}$ fixes the plane $\mathfrak{l}_{0}$.) Since $\Gamma$ is Zariski-dense, it follows that $\mathfrak{l}_{0}$ is an ideal in $\mathfrak{l}$. Let $\mathfrak{l}=\mathfrak{g}_{1} \oplus \mathfrak{g}_{2} \oplus \cdots \oplus \mathfrak{g}_{k}$ be the decomposition of $\mathfrak{l}$ into its simple Lie algebra summands. Hence $\mathfrak{l}_{\mathfrak{o}}=\mathfrak{g}_{i_{1}} \oplus \mathfrak{g}_{i_{2}} \oplus \cdots \oplus \mathfrak{g}_{i_{s}}$ for $i_{1}, \ldots, i_{s}$ distinct elements of $\{1, \ldots, k\}$.

Two cases arise:

Case $1 L_{0}$ is noncompact. Let $K \subset L$ be the maximal compact subgroup of $L$ and $X=L / K$ be the associated symmetric space. Then $X_{0}=L_{0} /\left(L_{0} \cap K\right)$ is the symmetric subspace of $L$ associated to the subgroup $L_{0}$. Further, $L_{0}$ is normal in $L$, since $\mathfrak{l}_{0}$ is an ideal in $\mathfrak{l}$ and $L_{0}$ is connected. Since $L_{0}$ is noncompact, its limit set is nonempty and since $L_{0}$ is normal, its limit set is all of $\partial X$ (This is essentially the only place where we really use the rank one assumption).

Using the associated Killing form [13] for instance, $X_{0}$ is a totally geodesic subspace of $X$. But from the previous paragraph, the limit set of $L_{0}$ is all of $\partial X$. Hence $X_{0}=X$ and $\overline{\operatorname{Comm}(\Gamma)}=L=\operatorname{Isom}(X)$.

Case $2 L_{0}$ is compact. Then $L_{0}$ fixes some point $x \in X$. Further, $L_{0}$ is normal in $L$, since $\mathfrak{l}_{0}$ is an ideal in $\mathfrak{l}$. Hence for any $g \in L$ and $l \in L_{0},\left(g l g^{-1}\right) g . x=g l . x=g . x$ and so $L_{0}$ fixes $g$. $x$. Hence $L_{0}$ fixes all $x \in X$. Therefore $L_{0}$ is trivial, since $L=\operatorname{Isom}(X)$.

As an immediate Corollary of Proposition 1.1, we have the following:

Corollary 1.2 Suppose $\Gamma$ is a Zariski-dense subgroup of a semisimple Lie group $L=\operatorname{Isom}(X)$ for $X$ a rank one symmetric space of noncompact type. Further suppose that the limit set $\Lambda_{\Gamma} \neq \partial X$. Then the commensurator $\operatorname{Comm}(\Gamma)$ of $\Gamma$ is discrete in $L$.

Proof The limit set $\Lambda_{\Gamma}$ is invariant under $\operatorname{Comm}(\Gamma)$. Hence if $\operatorname{Comm}(\Gamma)$ is dense in $L, \Lambda_{\Gamma}=\partial X$. Therefore by Proposition 1.1, $\operatorname{Comm}(\Gamma)$ is discrete in $L$.

Next, we generalize Proposition 1.1 to arbitrary semisimple $L=\operatorname{Isom}(X)$, by introducing an appropriate generalization of the notion of irreducibility. Motivated by the definition of irreducibility of lattices we propose the following. 
Definition 1.3 A Zariski dense subgroup $\Gamma$ of a semisimple Lie group $L$ will be called strongly irreducible if for any proper nontrivial normal subgroup $L_{0}$ of $L$, the projection of $\Gamma$ to the quotient $L / L_{0}$ is indiscrete.

Now, let $\Gamma$ be a strongly irreducible Zariski-dense subgroup of a semisimple Lie group $L=\operatorname{Isom}(X)$. As in Proposition 1.1 let $L_{0}$ be the connected component of the identity in $\overline{\operatorname{Comm}(\Gamma)}$. Suppose $L_{0} \neq\{1\}$. The quotient $\overline{\operatorname{Comm}(\Gamma)} / L_{0}$ is discrete. Since $\Gamma \subset \overline{\operatorname{Comm}(\Gamma)}$, it follows that $\overline{\operatorname{Comm}(\Gamma)}=L_{0}$. Since $\Gamma$ is Zariski-dense, $L_{0}=L$ and we have shown the following.

Proposition 1.4 Suppose $\Gamma$ is a strongly irreducible Zariski-dense subgroup of a semisimple Lie group $L=\operatorname{Isom}(X)$ for $X$ a symmetric space of noncompact type. Then the commensurator $\operatorname{Comm}(\Gamma)$ of $\Gamma$ is either discrete or dense in $L$.

The following is another simple generalization of Proposition 1.1.

Proposition 1.5 Suppose $\Gamma$ is a Zariski-dense subgroup of a semisimple Lie group $L=\operatorname{Isom}(X)$ for $X$ a symmetric space of noncompact type. If $\operatorname{Comm}(\Gamma)$ denotes the commensurator of $\Gamma$, then there exists a short exact sequence

$$
1 \rightarrow L_{0} \rightarrow \overline{\operatorname{Comm}(\Gamma)} \rightarrow Q \rightarrow 1
$$

where $L_{0}$ is a semisimple Lie subgroup of $L$ and $Q$ is discrete in $L / L_{0}$.

Proof As in the proof of Proposition 1.1, let $\overline{\operatorname{Comm}(\Gamma)}$ be the closure of $\operatorname{Comm}(\Gamma)$ in the Lie group $L$ and $L_{0}$ be the connected component of the identity in $\overline{\operatorname{Comm}(\Gamma)}$. If $L_{0}$ is trivial, then $\overline{\operatorname{Comm}(\Gamma)}$ is totally disconnected and hence $\operatorname{Comm}(\Gamma)$ is discrete.

Else, as before, let $\mathfrak{l}_{0}$ denote the Lie algebra of $L_{0}$ and $\mathfrak{l}$ denote the Lie algebra of $L$. Then $L_{0} \subset \overline{\operatorname{Comm}(\Gamma)}$ is normal and $\overline{\operatorname{Comm}(\Gamma)} / L_{0}=Q$ is discrete.

We now proceed to refine some of these results to higher rank semisimple Lie groups in the context of Zariski dense subgroups of $\operatorname{Isom}(X)$ acting on the flag variety or the Furstenberg boundary. This gives a geometric perspective on Proposition 1.5. Let $G$ be a semisimple Lie group, $K$ a maximal compact subgroup and $P$ a Borel subgroup. Then $G / P$ is called the Furstenberg boundary of the associated symmetric space $X=G / K$. See Benoist [2] for details. Limit sets of Zariski-dense subgroups $\Gamma$ of $G$ have been defined by Benoist [2] as in the rank one case. The limit set of the action of $\Gamma$ on $G / P$ will be denoted as $\Lambda(G / P, \Gamma)$. The following basic Proposition is due to Benoist, generalizing an analogous statement for $\mathrm{SL}_{n}(\mathbb{R})$ by Guivarc'h. 
Proposition 1.6 (Benoist [2]) Let $\Gamma \subset G$ be a Zariski dense subgroup. Then $\Lambda(G / P, \Gamma)$ is the unique minimal closed $\Gamma$-invariant subset $G / P$.

A semisimple Lie algebra is a direct sum of simple Lie algebras. A closed semisimple Lie subgroup $L_{0}$ of a Lie group $L$ will be called a (virtual) factor if its Lie algebra $\mathfrak{l}_{0}$ is a direct summand of the Lie algebra $\mathfrak{l}$ of $L$.

Proposition 1.6 will be essential in generalizing Corollary 1.2 to the following.

Proposition 1.7 Suppose $\Gamma$ is a Zariski-dense subgroup of a semisimple Lie group $L=\operatorname{Isom}(X)$ for $X$ a symmetric space of noncompact type. Further suppose that the limit set $\Lambda(G / P, \Gamma)$ is not invariant under any nontrivial semisimple (virtual) factor $L_{0}$. Then the commensurator $\operatorname{Comm}(\Gamma)$ of $\Gamma$ is discrete in $L$.

Proof As in the proof of Proposition 1.1, let $\overline{\operatorname{Comm}(\Gamma)}$ be the closure of $\operatorname{Comm}(\Gamma)$ in the Lie group $L$ and $L_{0}$ be the connected component of the identity in $\overline{\operatorname{Comm}(\Gamma)}$. If $L_{0}$ is trivial, then $\overline{\operatorname{Comm}(\Gamma)}$ is totally disconnected and hence $\operatorname{Comm}(\Gamma)$ is discrete.

Else, as before, let $\mathfrak{l}_{0}$ denote the Lie algebra of $L_{0}$ and $\mathfrak{l}$ denote the Lie algebra of $L$. Two cases arise:

Case $1 L_{0}$ is compact. Then exactly the same proof as Case 2 of Proposition 1.1 shows that $L_{0}$ is trivial.

Case $2 L_{0}$ is noncompact. $\operatorname{Comm}(\Gamma) \cap L_{0}=\Gamma_{0}$ is an infinite normal subgroup of $\operatorname{Comm}(\Gamma)$. Also, $\Gamma_{0}$ is Zariski-dense in $L_{0}$. Let $\Lambda_{\Gamma}$ and $\Lambda_{\Gamma_{0}}$ denote the limit sets of $\Gamma$ and $\Gamma_{0}$ respectively. Both are nonempty.

For every $g \in L, g . \Lambda_{\Gamma}$ is the limit set of $g . \Gamma$ and hence $g . \Gamma g^{-1}$. Since the limit set of a Zariski dense group is the unique minimal invariant set (Proposition 1.6), and since a finite index subgroup of a Zariski dense group is Zariski dense, $g . \Lambda_{\Gamma}=\Lambda_{\Gamma}$ for every $g \in \operatorname{Comm}(\Gamma)$, and hence for every $g \in \overline{\operatorname{Comm}(\Gamma)}$. In particular, $\Lambda_{\Gamma}$ is $L_{0}$-invariant. But by hypothesis $\Lambda_{\Gamma}$ is not invariant under any such $L_{0}$, a contradiction.

Therefore $\operatorname{Comm}(\Gamma)$ is discrete in $L$.

Remark 1.8 In terms of limit sets, invariance of $\Lambda_{\Gamma} \subset G / P$ under the action of a semisimple Lie subgroup $L_{0} \subset L$ means that $\Lambda_{\Gamma}=L_{0} / P_{0} \times \Lambda_{1}$, where

(1) $L=L_{0} \times L_{1}$ (at least virtually),

(2) $L_{0} / P_{0}$ is the Furstenberg boundary of $L_{0}$,

(3) $\Lambda_{1}$ is the limit set of the induced action of $\Gamma$ on the Furstenberg boundary $L_{1} / P_{1}$ of the semisimple $L_{1}$. 


\section{Relations on boundaries}

\subsection{Hyperbolicity and relative hyperbolicity}

Definition 2.1 For any geodesic metric space $(H, d)$, the hyperbolic cone $H^{h}$ is the metric space $H \times[0, \infty)=H^{h}$ equipped with a path metric $d_{h}$ obtained from two pieces of data :

(1) $d_{h, t}((x, t),(y, t))=2^{-t} d_{H}(x, y)$, where $d_{h, t}$ is the induced path metric on $H \times\{t\}$. Paths joining $(x, t),(y, t)$ and lying on $H \times\{t\}$ are called horizontal paths.

(2) $d_{h}((x, t),(x, s))=|t-s|$ for all $x \in H$ and for all $t, s \in[0, \infty)$, and the corresponding paths are called vertical paths.

For all $x, y \in H^{h}, d_{h}(x, y)$ is the path metric induced by the collection of horizontal and vertical paths.

Definition 2.2 Let $X$ be a proper (ie complete and locally compact) geodesic metric space and $\mathcal{H}$ be a collection of uniformly separated subsets of $X . X$ is said to be hyperbolic relative to $\mathcal{H}$ in the sense of Gromov, if the space $\mathcal{G}(X, \mathcal{H})$, obtained by attaching the hyperbolic cones $H^{h}$ to $H \in \mathcal{H}$ by identifying $(z, 0)$ with $z$ for all $H \in \mathcal{H}$ and $z \in H$, is a proper hyperbolic metric space. The collection $\left\{H^{h}: H \in \mathcal{H}\right\}$ is denoted as $\mathcal{H}^{h}$. The induced path metric on $\mathcal{G}(X, \mathcal{H})$ is also denoted by $d_{h}$.

A group $G$ is hyperbolic relative to a finite collection $H_{1}, \ldots, H_{k}$, if the Cayley graph $\Gamma$ of $G$ with respect to some finite generating set is hyperbolic relative to the collection $\mathcal{H}$ of translates of Cayley subgraphs of $H_{1}, \ldots, H_{k}$ (see Gromov [11], Farb [5] and Bowditch [3] for details on relative hyperbolicity). Then $\partial G=\partial \mathcal{G}(G, \mathcal{H})$ is called the (relative) hyperbolic (or Bowditch [3])-boundary of $G$, and $\widehat{G}=\mathcal{G}(G, \mathcal{H}) \cup \partial \mathcal{G}(G, \mathcal{H}$ ) is the compactification of $\partial \mathcal{G}(G, \mathcal{H})$. The set of distinct pairs of points on $\partial G$ will be denoted as $\partial^{2} G$.

Let $G$ be a (relatively) hyperbolic group. A fixed point on $\partial G$ of a hyperbolic element $g$ of $G$ is called a pole (See [11, Section 5.1]), and the pair of fixed points $\left(g_{-\infty}, g_{\infty}\right)$ is called a pole-pair.

Proposition 2.3 (Pole-pairs dense; Gromov [11, Section 5.1, page 136]) The collection of pairs $\left(g_{-\infty}, g_{\infty}\right)$ as $g$ ranges over hyperbolic elements of $G$ is dense in $\partial^{2} G$. 


\subsection{Cannon-Thurston maps}

Let $\left(X, d_{X}\right)$ be a proper hyperbolic metric space and $G$ be a (Gromov) hyperbolic group acting freely, properly discontinuously by isometries on $X$. Let $\Gamma$ be a Cayley graph of $G$ with respect to some finite generating set. By adjoining the Gromov boundaries $\partial X$ and $\partial G$ to $X$ and $\Gamma$, one obtains their compactifications $\hat{X}$ and $\widehat{G}$ respectively. Choose a basepoint $o \in X$. Let $i: \Gamma \rightarrow X$ denote an "inclusion map" mapping $g \in \Gamma$ to $g . o$ and an edge $[a, b]$ of $\Gamma$ to a geodesic in $X$ joining $a . o, b . o$. A Cannon-Thurston map $\hat{i}$ from $\widehat{G}$ to $\hat{X}$ is a continuous extension of $i$. The restriction of $\hat{i}$ to $\partial G$ will be denoted by $\partial i$. The map $\partial i$ induces a relation $\mathcal{R}_{\mathrm{CT}}$ on $\partial G$ where $x \sim y$ if $\partial i(x)=\partial i(y)$ for $x, y \in \partial G$.

Definition 2.4 A CT leaf $\lambda_{\mathrm{CT}}$ is a bi-infinite geodesic in $\Gamma$ whose endpoints are identified by $\partial i$.

Next, let $G$ be a relatively hyperbolic group, hyperbolic relative to a collection $H_{1}, \ldots, H_{k}$ of subgroups. Let, as before, $\left(X, d_{X}\right)$ be a hyperbolic metric space and $G$ act freely, properly discontinuously by isometries on $X$, such that each conjugate of $H_{i}$ fixes a unique point of $\partial X$. We thus think of each conjugate of $H_{i}$ acting on $X$ as a group of parabolic isometries. Let, as in Section 2.2, $i: \Gamma \rightarrow X$ denote the "inclusion map" mapping $g \in \Gamma$ to $g . o$ for some basepoint $o \in X$. Then $i$ induces a $\operatorname{map} i^{h}: \mathcal{G}(G, \mathcal{H}) \rightarrow X$.

A Cannon-Thurston map $\hat{i}$ from $\widehat{G}$ to $\hat{X}$ is a continuous extension of $i^{h}$. As before, the restriction of $\hat{i}$ to $\partial G$ will be denoted by $\partial i$ and the induced relation on $\partial G$ by $\mathcal{R}_{\mathrm{CT}}$.

Also, the set of distinct pairs of points identified by $\partial i$ will be denoted as $\mathcal{R}_{\mathrm{CT}}^{2}$, which is a subset of $\partial^{2}(G)$.

Lemma 2.5 $i: \Gamma \rightarrow X$ (or $i^{h}: \mathcal{G}(G, \mathcal{H}) \rightarrow X$ ) is a quasi-isometric embedding if and only if $\mathcal{R}_{\mathrm{CT}}^{2}=\varnothing$.

Proof If $i$ (or $i^{h}$ ) is a quasi-isometric embedding then $\partial i: \partial G \rightarrow \partial X$ is a homeomorphic embedding [11, Theorem 7.2H, page 189]. Hence $\mathcal{R}_{\mathrm{CT}}^{2}=\varnothing$.

Conversely, if $i$ (or $i^{h}$ ) is not a quasi-isometric embedding then there exist $a_{n}, b_{n} \in i(\Gamma)$ (or $i^{h}(\mathcal{G}(G, \mathcal{H}))$ ) such that the geodesic $\left[a_{n}, b_{n}\right]_{\Gamma} \subset \Gamma$ passes through $1 \in \Gamma$, but the geodesic $\left[a_{n}, b_{n}\right]_{X} \subset X$ joining $i\left(a_{n}\right), i\left(b_{n}\right)$ in $X$ lies outside $B_{n}(i(1))$, the $n$-ball about $i$ (1) in $X$. Assume (after subsequencing) that $a_{n} \rightarrow a_{\infty} \in \partial G$ and $b_{n} \rightarrow b_{\infty} \in \partial G$. It follows that $\partial i\left(a_{\infty}\right)=\partial i\left(b_{\infty}\right)$. Hence $a_{\infty} \sim b_{\infty}$ and $\mathcal{R}_{\mathrm{CT}}^{2} \neq \varnothing$.

Definition 2.6 $\mathcal{R}_{\mathrm{CT}}$ will be called trivial if $x \sim y$ for all $x, y \in \partial G$, and quasiconvex if $\mathcal{R}_{\mathrm{CT}}^{2}=\varnothing$. 


\subsection{Properties of the $\mathrm{CT}$ relation}

We assume in this subsection that $G$ is a (relatively) hyperbolic group acting freely, properly discontinuously by isometries on $\left(X, d_{X}\right)$, a hyperbolic metric space. Further we assume that a Cannon-Thurston map $\hat{i}$ from $\widehat{G}$ to $\hat{X}$ exists. The restriction of $\hat{i}$ to $\partial G$ is denoted by $\partial i$. Recall that $\partial i$ induces a relation $\mathcal{R}_{\mathrm{CT}}$ on $\partial G$ where $x \sim y$ if $\partial i(x)=\partial i(y)$ for $x, y \in \partial G$. Note that $\mathcal{R}_{\mathrm{CT}}$ is $G$-invariant. We investigate the basic properties of $\mathcal{R}_{\mathrm{CT}}$ now. Assume that $\mathcal{R}_{\mathrm{CT}}$ is nontrivial.

The following Lemma is a direct consequence of the continuity of $\hat{i}$.

Lemma 2.7 $\mathcal{R}_{\mathrm{CT}}$ is a closed relation on $\partial G$, ie if $x_{n} \sim y_{n}$ for sequences $\left\{x_{n}\right\},\left\{y_{n}\right\} \in$ $\partial G$ and $x_{n} \rightarrow x \in \partial G, y_{n} \rightarrow y \in \partial G$, then $x \sim y \in \partial G$. Further, $\mathcal{R}_{\mathrm{CT}}^{2}$ is a closed subset of $\partial^{2}(G)$.

Definition 2.8 $G$ is said to act on $X$ without accidental parabolics if for every hyperbolic element $g$ of $G$, its fixed points on $\partial X$ are distinct.

The next Lemma says that poles cannot lie in $\mathcal{R}_{\mathrm{CT}}$.

Lemma 2.9 Suppose $G$ acts on $X$ without accidental parabolics If $(x, y) \in \mathcal{R}_{\mathrm{CT}}$ and $x \neq y$, then $x$ cannot be a pole of $G$.

Proof By $G$-invariance of $\mathcal{R}_{\mathrm{CT}}$ we can assume that the CT-leaf $(x, y) \subset \Gamma$ passes through $1 \in \Gamma$. We argue by contradiction. If $x$ is a pole, there exists a hyperbolic element $g \in G$ such that $g_{\infty}=x$ and so $g$ acts on $X$ as a hyperbolic isometry. Then the infinite geodesic ray $[1, x) \subset \Gamma$ is a $C$-quasigeodesic in $X$ for some $C \geq 1$ since $G$ acts without accidental parabolics. Choosing a sequence of points $x_{n} \in[1, x) \subset(x, y)$ such that $x_{n} \rightarrow \infty$, note that $x_{n}^{-1} \cdot(x, y)$ and $x_{n}^{-1} \cdot[1, x)$ converge (up to subsequencing) to the same bi-infinite geodesic $(p, q) \subset \Gamma$. Hence $(p, q) \subset \Gamma$ is a $C$-quasigeodesic in $X$. In particular $\partial i(p) \neq \partial i(q)$. But, by Lemma $2.7(p, q) \in \mathcal{R}_{\mathrm{CT}}$, ie $\partial i(p)=\partial i(q)$. This is a contradiction.

Density The next Lemma proves density of orbits of cosets of $\mathcal{R}_{\mathrm{CT}}$ in the Hausdorff metric.

Lemma 2.10 Let $G$ be a (relatively) hyperbolic group acting on a hyperbolic metric space without accidental parabolics and admitting a Cannon-Thurston map. Assume that the relation $\mathcal{R}_{\mathrm{CT}}$ is nontrivial, ie not all points are in the same equivalence class. Let $K \subset \mathcal{R}_{\mathrm{CT}}$ be a coset (equivalence class) of the relation. Let $C_{c}(\partial G)$ denote the space of closed subsets of $\partial G$ with the Hausdorff metric. Then for all $x \in \partial G$, the singleton set $\{x\}$ is an accumulation point of $\{g . K: g \in G\}$. 
Proof Clearly, $K \subset \partial G$ is closed. By Proposition 2.3 and Lemma 2.9, $K$ is nowhere dense in $\partial G$ and, for all $x \in \partial G$, there exist a sequence $\left\{h_{n}\right\} \subset G$ such that

(1) the pole pair $\left(h_{n,-\infty}, h_{n, \infty}\right)$ of $h_{n}$ is a subset of $\partial G \backslash K$,

(2) $h_{n, \infty} \rightarrow x \in \partial G$ as $n \rightarrow \infty$.

By taking sufficiently large powers $h_{n}^{i_{n}}$ of $h_{n}$, we can ensure that $h_{n}^{i_{n}}(K)$ lies in an $\epsilon_{n}$ Hausdorff neighborhood of $h_{n, \infty}$, where $\epsilon_{n} \rightarrow 0$. Hence $h_{n}^{i_{n}}(K) \rightarrow\{x\} \in C_{c}(\partial G)$.

Remark 2.11 The assumption on absence of accidental parabolics is not too restrictive. In all cases of interest, subgroups $H$ of $G$ which become parabolic in $X$ will be regarded as elements of the collection $H_{1}, \ldots, H_{n}$ relative to which $G$ is hyperbolic.

\subsection{Continuity in uniform topology}

Let $\Lambda_{G} \subset \partial X$ denote the limit set of $G$, ie the collection of accumulation points in $\partial X$ of an(y) orbit of $G$ acting on $X$. The existence of a Cannon-Thurston map ensures that $\partial i: \partial G \rightarrow \Lambda_{G}$ is a quotient map, where preimages of $\partial i$ are given by elements of $\mathcal{R}_{\mathrm{CT}}$. Let $\partial i_{c}: C_{c}(\partial G) \rightarrow C_{c}\left(\Lambda_{G}\right)$ be the map induced by $i_{c}$ from $\mathcal{R}_{\mathrm{CT}}$-saturated compact subsets of $\partial G$ to compact subsets of $\Lambda_{G}$ both equipped with the Hausdorff metric. Suppose that $f$ is a homeomorphism of $\partial G$ that preserves the cosets of $\mathcal{R}_{\mathrm{CT}}$, ie for any coset $K \in \mathcal{R}_{\mathrm{CT}}, f(K)$ is also a coset of $\mathcal{R}_{\mathrm{CT}}$. Then the quotient map from $\Lambda_{G}$ to itself induced by $f$ will be denoted by $\bar{f}$.

Proposition 2.12 Suppose that $\mathcal{R}_{\mathrm{CT}}$ is nontrivial. Let $f_{n}$ be a sequence of homeomorphisms of $(\partial G, d)$ that preserves the cosets of $\mathcal{R}_{\mathrm{CT}}$, where $d$ denotes some visual metric. Let $\bar{f}_{n}$ denote the induced homeomorphisms of $\Lambda_{G}$. If $f_{n} \rightarrow$ id in the uniform topology on Homeo( $(\partial G)$ then $\overline{f_{n}} \rightarrow$ id in the uniform topology on $\operatorname{Homeo}\left(\Lambda_{G}\right)$. Conversely, if $\bar{f}_{n} \rightarrow$ id in the uniform topology on $\operatorname{Homeo}\left(\Lambda_{G}\right)$ then for every pole $p \in \partial G, d\left(p, f_{n}(p)\right) \rightarrow 0$. More generally, if $\{p\}$ is a coset of $\mathcal{R}_{\mathrm{CT}}, d\left(p, f_{n}(p)\right) \rightarrow 0$.

Proof The forward direction follows from the definition of quotient maps. Conversely, suppose $\bar{f}_{n} \rightarrow$ id in the uniform topology on Homeo $\left(\Lambda_{G}\right)$ but $f_{n}$ does not converge to the identity map in the uniform topology on $\operatorname{Homeo}(\partial G)$. Suppose that there exists (up to subsequencing) an $\epsilon>0$ and a pole $x \in \partial G$ (or a point $x$ such that $\{x\}$ is a coset of $\left.\mathcal{R}_{\mathrm{CT}}\right)$ such that for all $n \in \mathbb{N} d\left(f_{n}(x), x\right)>\epsilon$.

Then there exists $\eta<\epsilon / 10$, say, such that $d_{H}\left(B_{\eta}(x), f_{n}\left(B_{\eta}(x)\right)\right)>\eta$ where $d_{H}$ denotes the Hausdorff metric on $C_{c}(\partial G)$ and $B_{\eta}(w)$ denotes a closed ball of radius $\eta$ centered at $w$. 
Assume after subsequencing that $f_{n}(x) \rightarrow y \in \partial G$. Then $x \sim y$, since $\overline{f_{n}} \rightarrow$ id. But $x \neq y$.

This contradicts Lemma 2.9, if $x$ is a pole (or, more generally, if $\{x\}$ is a coset of $\mathcal{R}_{\mathrm{CT}}$ ).

\section{Commensurators of Kleinian groups}

In this section we prove discreteness of commensurators for finitely generated, discrete, Zariski dense, infinite covolume Kleinian groups.

\subsection{Surface groups}

It follows from the Scott Core Theorem [26] that any finitely generated Kleinian group $H$ is the fundamental group of a compact 3 manifold with boundary. Further, a geometrically finite group $H_{\mathrm{gf}}$ can be chosen abstractly isomorphic to $H$ such that the isomorphism preserves parabolics (see McCullough, Miller and Swarup [20], McCullough [19] and Feighn and McCullough [7] for relative versions of the Scott core Theorem). Hence abstractly $G$ is hyperbolic relative to its parabolic subgroups (see Farb [5] for instance). The convex core of $\mathbb{H}^{3} / H$ will be denoted by $M$ and the convex core of $\mathbb{H}^{3} / H_{\mathrm{gf}}$ will be denoted by $K$, so that the inclusion $K \subset M$ is a homotopy equivalence. We proved the following theorem:

Theorem 3.1 [23;22] Let $G$ be a finitely generated Kleinian group. Then $G$ admits a Cannon-Thurston map from the (relative) hyperbolic boundary of $G$ to its limit set. Further, the Cannon-Thurston map from the (relative) hyperbolic boundary of $G$ to its limit set identifies precisely the endpoints of leaves of the ending laminations.

By Corollary 1.2, it suffices to consider the case where all ends of $M$ are degenerate as these are precisely the manifolds that come from Kleinian groups with empty domain of discontinuity. Our study of $\operatorname{Comm}(H)$ splits into two cases:

Case I Some component of the boundary $\partial K$ of $K$ is an incompressible geometrically finite surface.

Case II $K$ is topologically a compression body whose lower boundary is a (possibly empty) collection of tori.

This dichotomy stems from the fact that if some boundary component $Q$ of $K$ is incompressible relative to rank one cusps, then some other component of $\partial K$ is an incompressible geometrically finite surface unless $K$ is topologically a compression 
body whose lower boundary is a (possibly empty) collection of tori. This follows from standard 3-manifold topology [14] by choosing a maximal collection of disjoint homotopically distinct compressing disks with boundary curves on $Q$. In particular, if some maximal cusp of $K$ is of rank one, we are in Case I.

We deal with Case I in this subsection and indicate the modifications for Case II in the next subsection.

Lemma 3.2 Let $M, K$ be as above. Let $\Sigma \subset \operatorname{Bdy}(K)$ be a finite area surface component of the boundary of $K$ that is incompressible away from cusps. Further suppose that the ideal (relative) hyperbolic boundary $\partial(\widetilde{\Sigma})=S^{1}$ is a proper subset of the (relative) hyperbolic boundary $\partial(\widetilde{K})$.

Let $\bar{f}_{n} \in \operatorname{Comm}(H)$ be a sequence of commensurators converging to the identity in Isom $\left(\mathbb{H}^{3}\right)$ and let $f_{n}$ be the induced homeomorphisms on the (relative) hyperbolic boundary $\partial(\tilde{K})$. Then $f_{n} \rightarrow \operatorname{Id} \in \operatorname{Homeo}\left(S^{1}\right)$.

Proof Suppose $f_{n}$ does not converge to the identity map $\operatorname{Id} \in \operatorname{Homeo}\left(S^{1}\right)$. Then by Proposition 2.12 it suffices to show that there exists (up to subsequencing) a pole $p \in \partial(\widetilde{K})$ and an $\epsilon>0$ such that $d\left(f_{n}(p), p\right) \geq \epsilon$ for all $n$, where $d$ is a visual metric on $\partial(\widetilde{K})$.

Since $f_{n}$ does not converge to the identity, we may assume (after passing to a further subsequence if necessary) that there exists a translate $T(=g . \partial(\widetilde{\Sigma}))$ of $\partial(\widetilde{\Sigma})$ such that $f_{n}(T) \neq T$ for all $n$. Since $T$ has a definite visual diameter and the poles of $g \rho\left(\pi_{1}(\Sigma)\right) g^{-1}$ are dense in $g . \partial(\widetilde{\Sigma})$, there exists a pole $p \in g . \partial(\widetilde{\Sigma})$ and an $\epsilon>0$ such that $d\left(f_{n}(p), p\right) \geq \epsilon$ for all $n$

It remains to deal with doubly degenerate surface groups. By passing to a subgroup of index at most two in the commensurator group if necessary, we can assume without loss of generality that ends of doubly degenerate groups are preserved. Thus, in any case, for a sequence of commensurators $g_{n}$ converging to the identity an end corresponding to a strictly type-preserving representation $\rho: \pi_{1}(S) \rightarrow \operatorname{Isom}\left(\mathbb{H}^{3}\right)$ is "preserved". The image $\rho\left(\pi_{1}(S)\right)$ is simply the subgroup of $H$ corresponding to an incompressible end of $M$. By Theorem 3.1 we may assume that $\left\{g_{n}\right\}$ preserves the relation $\mathcal{R}_{\mathrm{CT}}$ corresponding to the Cannon-Thurston map for the end. Let $\mathcal{L}$ be the ending lamination whose endpoints are identified by the Cannon-Thurston map.

Claim 3.3 Let $\bar{f}_{n} \in \operatorname{Comm}(H)$ be a sequence of commensurators converging to the identity in $\operatorname{Isom}\left(\mathbb{H}^{3}\right)$ and let $f_{n}$ be the induced homeomorphisms on the (relative) hyperbolic boundary $\partial \pi_{1}(S)\left(=S^{1}\right)$ of the group $\pi_{1}(S)$. Then $f_{n} \rightarrow \operatorname{Id} \in \operatorname{Homeo}\left(S^{1}\right)$. 
Proof of Claim Suppose $f_{n}$ does not converge to the identity map $\operatorname{Id} \in \operatorname{Homeo}\left(S^{1}\right)$. Then by Proposition 2.12 it suffices to show that there exists (up to subsequencing) a pole $p \in S^{1}$ and an $\epsilon>0$ such that $d\left(f_{n}(p), p\right) \geq \epsilon$ for all $n$, where $d$ is the usual visual metric on $S^{1}$.

Since $f_{n}$ does not converge to the identity, we may assume (after passing to a further subsequence if necessary) that there exists an ideal polygon $\Delta$ such that

(a) the sides of $\Delta$ are leaves of the ending lamination $\mathcal{L}$,

(b) $\left.f_{n}\right|_{\Delta} \neq$ Id for all $n$.

Then there exist ideal adjacent vertices $A, B$ of $\Delta$ such that $f_{n}(A) \neq A$ and $f_{n}(B) \neq B$ for all $n$. Let $\Delta_{1}$ be another polygon whose sides lie in $\mathcal{L}$ and whose endpoints lie in the arc $(A B)$ of the circle $S^{1}$ that contains no other ideal points of $\Delta$. Also let $A_{1}, B_{1}$ be adjacent vertices of $\Delta_{1}$ such that $\left(A_{1} B_{1}\right) \subset(A B)$. See Figure 1 .

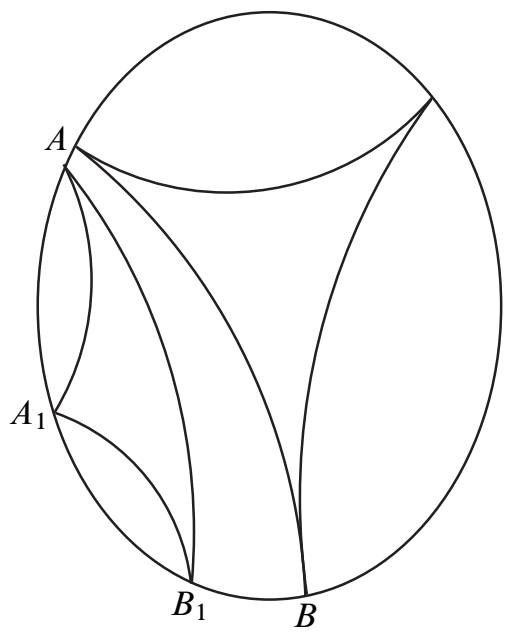

Figure 1

Since $f_{n}(A) \neq A$ and $f_{n}(B) \neq B$ for all $n$ it follows that any pole $p$ in the arc $\left(A_{1} B_{1}\right)$ is moved at least $\epsilon=\min \left(d\left(A, A_{1}\right), d\left(A, B_{1}\right), d\left(B, A_{1}\right), d\left(B, B_{1}\right)\right)$ by $f_{n}$ for all $n$. This proves the Claim.

We are finally in a position to prove the following.

Theorem 3.4 Let $G$ be a finitely generated Kleinian group such that $G$ is Zariski dense in $\mathrm{PSL}_{2}(\mathbb{C})$ and $\mathbb{H}^{3} / G$ has infinite volume. Further, suppose that some end of $M=\mathbb{H}^{3} / G$ is incompressible. Then the commensurator $\operatorname{Comm}(G)$ of $G$ is discrete in $\mathrm{PSL}_{2}(\mathbb{C})$. 
Proof As above, suppose $\bar{f}_{n} \in \operatorname{Comm}(H)$ be a sequence of commensurators converging to the identity in Isom $\left(\mathbb{H}^{3}\right)$ and let $f_{n}$ be the induced homeomorphisms on the (relative) hyperbolic boundary $\partial \pi_{1}(S)\left(=S^{1}\right)$ of the group $\pi_{1}(S)$. Then by Claim 3.3 it follows that for any ideal polygon $\Delta$ with boundary in the ending lamination there exists $N=N(\Delta)$ such that $f_{n}$ fixes all the vertices of $\Delta$ for all $n \geq N$. Let $z_{\Delta} \in S_{\infty}^{2}$ denote the common image of the ideal vertices of $\Delta$ under the Cannon-Thurston map of Theorem 3.1. The collection of translates of $z_{\Delta}$ is dense in $S^{2}$. Choose ideal polygons $\Delta_{1}, \ldots, \Delta_{4}$ such that the collection $\left\{z_{\Delta_{1}}, \ldots, z_{\Delta_{4}}\right\}$ is in general position on $S_{\infty}^{2}$, ie not all lying on the same circle. Then for all $n \geq \max _{i=1 \cdots 4}\left\{N\left(\Delta_{i}\right)\right\}, \bar{f}_{n}=\mathrm{Id}$. The Theorem follows.

Theorem 3.4 was proven by Leininger, Long and Reid [16] under the additional assumption that $G$ is nonfree and without parabolics.

\subsection{Compressible core}

It remains to prove the analogue of Theorem 3.4 in Case II, ie when the (relative) core $K$ is topologically a compression body whose lower boundary is a (possibly empty) collection of tori. As in the previous subsection, $H$ will denote the Kleinian group. The associated manifold $M=K \cup E$, where $E$ is homeomorphic to $S \times[0, \infty)$ and $E \cap K=S \times\{0\}$. Further $E$ is a simply degenerate end. Let $\sigma$ be a homotopically essential simple closed curve on the boundary $\operatorname{Bdy}(K)$ of $K$.

Background In [21], Minsky constructs a model for the simply degenerate end $E$. We consider the family $\mathcal{F}$ of pleated surfaces exiting the end $E$ and let $\tilde{\mathcal{F}}$ be the collection of lifts of $\mathcal{F}$ to the universal cover $\widetilde{M}$ of $M$. The elements of $\widetilde{\mathcal{F}}$ are lifts $\widetilde{\Sigma}$ of pleated surfaces $\Sigma$ in $E$.

For any $g \in \operatorname{Comm}(H), H_{g}=g^{-1} H g \cap H$ is of finite index in $H$. Hence $M_{g}=$ $\mathbb{H}^{3} / H_{g}$ is a finite-sheeted cover of $M$. Let $\mathcal{F}_{g}$ denote the lifts of elements of $\mathcal{F}$ to $M_{g}$. Since $\sigma \subset \operatorname{Bdy}(K)=\operatorname{Bdy}(E)$ is homotopically trivial in $K$, any loop in $E$ freely homotopic to $\sigma$ in $E$ lifts isometrically to loops in $\widetilde{M}$ as well as in $M_{g}$. Denote by $\sigma_{\alpha} \subset \Sigma_{\alpha}$ the unique geodesic representative in the homotopy class of $\sigma$ in $E$ carried by the pleated surface $\Sigma_{\alpha} \in \mathcal{F}$.

In [22] we constructed the following.

(1) A subcollection $\left\{\Sigma_{\beta}, \beta \in \mathcal{B}\right\}$ of the collection $\mathcal{F}$.

(2) An auxiliary Gromov hyperbolic metric $d_{G}$ on $\widetilde{M}$ called the graph metric. 
(3) Let $D_{\sigma}$ denote a disk bounded by (a lift of) $\sigma$ in $\tilde{K}$. We also constructed a quasidisk $A_{\sigma}=\left(D_{\sigma} \cup_{\beta \in \mathcal{B}} \sigma_{\beta}\right)$ such that $A_{\sigma}$ is quasiconvex in $\left(\widetilde{M}, d_{G}\right)$. This construction is natural with respect to taking covers (finite or infinite) as $\sigma$ bounds in $K$. Hence any cover of $K$ contains isometric copies of $A_{\sigma}$. Since $A_{\sigma}$ is quasiconvex in $\left(\widetilde{M}, d_{G}\right)$, the limit set $\partial A_{\sigma}$ is a proper subset of $\mathbb{S}_{\infty}^{2}$ with diameter greater than zero.

(4) It is also shown in [22] that if $h \rightarrow \infty$ in $\Gamma_{H}$, then the visual diameter of $h . \partial A_{\sigma} \subset \mathbb{S}_{\infty}^{2}$ tends to zero. In particular, the collection $\left\{h . \partial A_{\sigma}\right\}$ is discrete in $\mathbb{S}_{\infty}^{2}$

We therefore conclude:

Lemma 3.5 Let $g_{n} \in \operatorname{Comm}(H)$ such that $g_{n} \rightarrow \mathrm{Id}$. Then for any $h_{1}, \ldots h_{k} \in H$, there exists $N>0$ such that $g_{n} .\left(h_{i} . \partial A_{\sigma}\right)=h_{i} . \partial A_{\sigma}$ for all $i=1, \ldots, k$ and $n \geq N$.

Remark 3.6 We may think of the collection $\left\{h . \partial A_{\sigma}\right\}$ as an $H$-invariant pattern on $\mathbb{S}_{\infty}^{2}$ along the lines of [24].

The last step of the argument is similar to Theorem 3.4. Let $\Delta$ be an ideal polygon contained in $\operatorname{Bdy}(\widetilde{K})$, whose sides are leaves of the ending lamination (thought of as an element of the Masur domain).

Choose a collection of lifts $\sigma_{1}, \ldots, \sigma_{m}$ of $\sigma$ to $\tilde{K}$ such that the following holds:

(1) The associated compressing disks $D_{1}, \ldots, D_{m}$ are the corresponding lifts of the disk $D$ bounded by $\sigma$ in $K$.

(2) The boundary of $\left(\tilde{K} \backslash \bigcup_{i=1, \ldots, m} D_{i}\right)$ consists of one bounded piece $\Sigma_{b}$ homeomorphic to a sphere with finitely many holes and a finite collection of unbounded components.

(3) Consider the singular foliation associated to the ending lamination. Then the polygon $\Delta$ naturally corresponds to an $l$-prong $\mathcal{P}-$ a union of $l$ geodesic rays starting at a common point $p \in \operatorname{Bdy}(\tilde{K})$. $p$ will be called the base of the $l$-prong $\mathcal{P}$. We assume that $p$ lies in the interior of $\Sigma_{b}$.

Let $A_{1}, \ldots, A_{m}$ be the quasidisks associated to $D_{1}, \ldots, D_{m}$. Let $g_{n} \in \operatorname{Comm}(H)$ such that $g_{n} \rightarrow \mathrm{Id}$. Then, by Lemma 3.5, there exists $N>0$ such that $g_{n} \cdot\left(\partial A_{i}\right)=\partial A_{i}$ for all $n \geq N$.

Since any commensurator of $G$ induces a homeomorphism of one finite sheeted cover of $K$ to another, it moves $\widetilde{K}$ to within a bounded Hausdorff distance of itself in $\widetilde{M}$. Hence we can isotope the image to $\widetilde{K}$ by a bounded isotopy. 
After a bounded isotopy, we may assume that $g_{n}\left(D_{i}\right)=D_{i}$ for $i=1 \cdots m$ and for all $n \geq N$. Hence the base $p$ of the $l$-prong $\mathcal{P}$ is moved to some point in $\Sigma_{b}$ by $g_{n}$ for all $n \geq N$.

However, there exist only finitely many $l$-prongs $(l>2)$ whose base lies in $\Sigma_{b}$. Also $g_{n}\left(D_{i}\right)$ can be isotoped to $D_{i}$ by a bounded isotopy of $\widetilde{M}$. Hence there exists $M \geq N$ such that $g_{n}(\mathcal{P})$ is homotopic to $\mathcal{P}$ by a bounded homotopy for all $n \geq M$.

As in Claim 3.3 let $f_{n}$ be the homeomorphisms of the (relative) hyperbolic boundary $\partial \widetilde{K}$ induced by $g_{n}$. Then for all $n \geq M, f_{n}(x)=x$ for all ideal endpoints $x$ of $\mathcal{P}$.

Let $q: \partial H \rightarrow \Lambda_{H}$ be the Cannon-Thurston map of Theorem 3.1 from the relative hyperbolic boundary of $H$ to its limit set $\Lambda_{H}$. Then $g_{n}(q(x))=q(x)$ for all $n \geq M$.

Choose four prongs $\mathcal{P}_{1}, \ldots, \mathcal{P}_{4}$ and ideal endpoints $x_{1}, \ldots, x_{4}$ such that the images $q\left(x_{i}\right)$ are in general position on $\mathbb{S}_{\infty}^{2}$. Then there exists $M_{1} \geq M$ such that $g_{n}\left(q\left(x_{i}\right)\right)=$ $q\left(x_{i}\right)$ for all $n \geq M_{1}$. Therefore $g_{n}=$ Id for all $n \geq M$.

We have finally proved the following.

Theorem 3.7 Let $G$ be a finitely generated Kleinian group such that $G$ is Zariski dense in $\mathrm{PSL}_{2}(\mathbb{C})$ and $\mathbb{H}^{3} / G$ has infinite volume. Then the commensurator $\operatorname{Comm}(G)$ of $G$ is discrete in $\mathrm{PSL}_{2}(\mathbb{C})$.

Using the Thurston-Canary covering theorem [4], Theorem 3.7 can be strengthened a la Leininger, Long and Reid [16] to show that unless $G$ corresponds to a fiber subgroup of a 3-manifold fibering over the circle, then, in fact, $[\operatorname{Comm}(G): G]<\infty$. We discuss this below.

Let $g \in \operatorname{Comm}(G)$ be a commensurator of $G$. Then the group $G_{1}=\langle G, g\rangle$ is a finitely generated discrete Kleinian group. Let $M=\mathbb{H}^{3} / G$ and $M_{1}=\mathbb{H}^{3} / G_{1}$. If $M$ is an infinite cover of $M$, ie if $\left[G_{1}: G\right]=\infty$, then the Thurston-Canary covering theorem [4] implies that $G$ is virtually a fiber subgroup of the finite-volume 3-manifold group $G_{1}$, ie $M_{1}$ has a finite sheeted cover that fibers over the circle with fiber subgroup of finite index in $G$. Else, inductively, for all $g_{1}, \ldots, g_{k} \in \operatorname{Comm}(G)$, if we denote $G_{k}=\left\langle G, g_{1}, \ldots, g_{k}\right\rangle$, then $\left[G_{k}: G\right]<\infty$. Let $M_{k}=\mathbb{H}^{3} / G_{1}$. Again, by the ThurstonCanary covering theorem [4], every end of $M_{k}$ is covered by an end of $M$. Since $M$ has a finite number of ends, we may assume after passing to a subsequence, that all the $M_{k}$ have the same number of ends. Since each end of $M_{k}$ is homeomorphic to $S \times[0, \infty)$ for some finite volume hyperbolic surface $S$ and since each $S$ can cover only finitely many hyperbolic surfaces, it follows that the sequence $M_{k}$ must stabilize. We have thus shown the following. 
Theorem 3.8 Let $G$ be a finitely generated Kleinian group such that $G$ is Zariski dense in $\operatorname{PSL}_{2}(\mathbb{C})$ and $\mathbb{H}^{3} / G$ has infinite volume. Then $[\operatorname{Comm}(G): G]<\infty$ unless $G$ is virtually a fiber subgroup, in which case $\operatorname{Comm}(G)$ is the fundamental group of a virtually fibered finite volume hyperbolic 3-manifold.

Finally, a note of caution: Just the existence of Cannon-Thurston maps is not sufficient to ensure discreteness of commensurators. A nonuniform arithmetic lattice in $\mathbb{H}^{2}$ has dense commensurator. However, work of Floyd [8] ensures the existence of CannonThurston maps in this situation. Thus the proof of Theorem 3.7 implicitly uses the following fact in an essential way:

Any polygon whose sides are leaves of an ending lamination has more than two sides.

This is exactly what goes wrong for nonuniform lattices in $\mathbb{H}^{2}$ and prevents us from using the separation arguments that go into the proof of Theorem 3.7.

\subsection{Examples, applications and questions}

We give an example to show that certain naive analogues of Theorem 3.8 cannot be expected in higher dimensions. The examples of fiber groups in Theorem 3.8 have empty domain of discontinuity. We first furnish an example that illustrates: Domain of discontinuity nonempty does not imply $[\operatorname{Comm}(\Gamma): \Gamma]<\infty$

Example of a group $\Gamma$ with $\Lambda_{\Gamma}$ a proper subset of $\partial X$, but $[\operatorname{Comm}(\Gamma): \Gamma]=\infty$ Let $M$ be a finite volume hyperbolic 3-manifold fibering over the circle and admitting a totally geodesic embedded incompressible surface $\Sigma$. Let $\rho: \pi_{1}(M) \rightarrow \operatorname{Isom}\left(\mathbb{H}^{3}\right)$ be the associated representation. Let $\rho^{\prime}: \pi_{1}(M) \rightarrow \operatorname{Isom}\left(\mathbb{H}^{4}\right)$ be $\rho$ composed with the embedding of $\operatorname{Isom}\left(\mathbb{H}^{3}\right)$ in $\operatorname{Isom}\left(\mathbb{H}^{4}\right)$. Then a bending deformation of $\rho^{\prime}$, keeping $\rho^{\prime}\left(\pi_{1}(\Sigma)\right.$ fixed gives a new discrete faithful representation $\rho^{\prime \prime}: \pi_{1}(M) \rightarrow \operatorname{Isom}\left(\mathbb{H}^{4}\right)$ with Zariski dense image and such that $\rho^{\prime \prime}\left(\pi_{1}(M)\right)=G$ is quasiconvex. Hence $\Lambda_{G} \neq \partial \mathbb{H}^{4}$. Now, let $F$ be the fiber of $M$. Then $\rho^{\prime \prime}\left(\pi_{1}(F)\right)=H$ is normal in $G$ and hence $\Lambda_{H}=\Lambda_{G} \neq \partial \mathbb{H}^{4}$. By Corollary 1.2, $\operatorname{Comm}(H)$ is discrete. But $G$ normalizes $H$. Therefore $G \subset \operatorname{Comm}(H)$. Hence $[\operatorname{Comm}(H): H]=\infty$.

Absence of examples in dimension 4 We indicate that a naive generalization of Theorem 3.8 cannot exist in dimension 4. In fact, in [12] Gromov comments:

Nonarithmetic $\Gamma$ are especially plentiful for $n=3$ by Thurston's theory and often have unexpected features, eg some $V=\mathbb{H}^{3} / \Gamma$ fiber over $S^{1}$ (which is hard to imagine ever happening for large $n$ ). 
The next Proposition provides some justification.

Proposition 3.9 Let $M$ be a closed aspherical four manifold fibering over the circle with fiber $F$. Then $\pi_{1}(M)$ cannot be Gromov-hyperbolic. In particular $M$ cannot be a rank one locally symmetric space. More generally if $M$ is a closed aspherical $n$-manifold fibering over an (n-3)-manifold with infinite fundamental group, then $\pi_{1}(M)$ cannot be Gromov-hyperbolic.

Proof Observe that $F \times \mathbb{R}$ covers $M$ and $\widetilde{F} \times \mathbb{R}=\widetilde{M}$ is aspherical. Hence $\pi_{i}(F)=0$ for all $i>1$. Hence $F$ is a closed aspherical 3-manifold. Suppose $\pi_{1}(M)$ is Gromovhyperbolic. Then $\pi_{1}(M)$ cannot contain a copy of $\mathbb{Z} \oplus \mathbb{Z}$. Hence $F$ is a closed aspherical atoroidal 3-manifold with infinite fundamental group. By Perelman's Geometrization Theorem, it follows that $F$ admits a hyperbolic structure. Hence $\operatorname{Out}\left(\pi_{1}(F)\right)$ is finite.

But $M=(F \times I) / \phi$ for a diffeomorphism $\phi$ inducing an infinite order element of $\operatorname{Out}\left(\pi_{1}(F)\right)$ as otherwise $\pi_{1}(M)$ would contain a copy of $\mathbb{Z} \oplus \mathbb{Z}$. This is a contradiction.

The same argument gives the more general conclusion in the statement of the Proposition.

Remark 3.10 The Chern-Gauss-Bonnet formula proves that an even dimensional hyperbolic manifold cannot fiber over the circle as such a manifold must have Euler characteristic zero.

An alternate way of increasing fibered faces An application of the ideas developed in this paper is an alternate argument (cf Long and Reid [17] and Agol [1]) for increasing the number of fibered faces of an arithmetic hyperbolic 3-manifold fibering over the circle.

Let $N$ be a finite volume arithmetic hyperbolic 3-manifold fibering over the circle. Equip $N$ with the suspension flow of the associated pseudo-Anosov monodromy. A theorem of Fried [6] says the following:

Theorem (Fried) Let $F_{1}$ and $F_{2}$ be two embedded incompressible connected surfaces in a fibered 3-manifold $M$. Then $F_{1}$ and $F_{2}$ lie in the same fiber face of the unit Thurston norm ball if and only the flow lines of the associated suspension pseudo-Anosov flows are isotopic. 
Take a stable polygon $\mathcal{P}$ (of more than two, possibly infinitely many sides, as in the punctured case) on a lift of the fiber $\widetilde{F} \subset \widetilde{F}$. Flow it forward and backward under the suspension flow to obtain a set $S(\mathcal{P})$. If $F$ is a punctured surface and $\mathcal{P}$ has infinitely many sides, adjoin a copy of the horoball $\mathcal{B}$ which has the same common horospherical base-point at infinity as $\mathcal{P}$. By [25], $\mathcal{B} \cup S(\mathcal{P})$ is a quasiconvex subset of $\mathbb{H}^{3}$. Let $\partial \mathcal{P}$ denote the limit set of $\mathcal{B} \cup S(\mathcal{P})$. Then the collection of translates $\left\{g . \partial \mathcal{P}: g \in \rho\left(\pi_{1}(S)\right)\right\}$ is a discrete collection of sets in the Hausdorff topology on $\mathbb{S}_{\infty}^{2}$.

This collection is a discrete pattern [24] which is an invariant of the fiber face of the unit Thurston norm ball, ie $F_{1}$ and $F_{2}$ lie in the same fiber face of the unit Thurston norm ball if and only if they give rise to the same invariant collection of translates $\left\{g . \partial \mathcal{P}: g \in \rho\left(\pi_{1}\left(F_{i}\right)\right)\right\}$.

Since the 3-manifold $N$ is arithmetic, $\operatorname{Comm}\left(\pi_{1}(N)\right)$ is dense. Therefore the $\operatorname{Comm}\left(\pi_{1}(N)\right)$-orbit of $\partial \mathcal{P}$ is not discrete. Hence, as in [1, Theorem 7.1] or [17], intersecting $\pi_{1}(N)$ with finitely many $g \pi_{1}(N) g^{-1}$ for appropriate $g \in \operatorname{Comm}\left(\pi_{1}(N)\right)$ one can increase the number of fiber faces arbitrarily.

Questions Together, Proposition 1.7 and Theorem 3.7 prove discreteness of commensurators for all known examples of finitely presented, Zariski dense, infinite covolume discrete subgroups of $\operatorname{Isom}(X)$ for $X$ a symmetric space of noncompact type. We therefore venture the following:

Question 3.11 If $\Gamma$ is a Zariski dense infinite covolume subgroup of $\operatorname{Isom}(X)$ for an irreducible symmetric space $X$ of noncompact type, is $\operatorname{Comm}(\Gamma)$ discrete?

For finitely presented groups, Question 3.11 subsumes the following (by Proposition 1.7 and Theorem 3.7).

Question 3.12 If $\Gamma$ is a finitely presented, infinite covolume, Zariski dense subgroup of $\operatorname{Isom}(X)$ for an irreducible symmetric space $X \neq \mathbb{H}^{3}$ of noncompact type, can the limit set be all of the Furstenberg boundary?

Misha Kapovich [15] has an example of a finitely generated but not finitely presented normal subgroup of a lattice in $\mathbb{C} \mathbb{H}^{2}$. Normality ensures that the limit set is all of the visual boundary.

If we leave the domain of finitely generated groups, then there do exist examples to which Question 3.11 applies.

Question 3.13 Let $\Gamma_{0}$ be an infinite index normal subgroup of a rank one (possibly arithmetic) lattice $\Gamma$. Is $\operatorname{Comm}\left(\Gamma_{0}\right)$ discrete? For instance $\Gamma_{0}$ could be the commutator subgroup $[\Gamma, \Gamma]$ when $H_{1}(\Gamma, \mathbb{Q}) \neq 0$. 
A particularly simple instance when even the special case of Question 3.13 seems unknown is when $\Gamma \subset S L_{2}(\mathbb{R})$ is a congruence subgroup of $\mathrm{SL}_{2}(\mathbb{Z})$.

Acknowledgments I would like to thank Kingshook Biswas, Tom Church, Pranab Sardar and T N Venkataramana for helpful email correspondence and discussions. I would also like to thank Alan Reid for pointing out errors and omissions in an earlier version of this paper. This work was done in part when the author was visiting Universite Paris-Sud (Orsay) under the ARCUS Indo-French programme. Research partly supported by a CEFIPRA project grant.

\section{References}

[1] I Agol, Criteria for virtual fibering, J. Topol. 1 (2008) 269-284 MR2399130

[2] Y Benoist, Propriétés asymptotiques des groupes linéaires, Geom. Funct. Anal. 7 (1997) 1-47 MR1437472

[3] B Bowditch, Relatively hyperbolic groups, Preprint (1999) Available at http:// www. warwick.ac.uk/ masgak/papers/bhb-relhyp.pdf

[4] RD Canary, A covering theorem for hyperbolic 3-manifolds and its applications, Topology 35 (1996) 751-778 MR1396777

[5] B Farb, Relatively hyperbolic groups, Geom. Funct. Anal. 8 (1998) 810-840 MR1650094

[6] A Fathi, F Laudenbach, V Poenaru, editors, Travaux de Thurston sur les surfaces, Astérisque 66-67, Soc. Math. France (1979) MR568308 Séminaire Orsay, With an English summary

[7] M Feighn, D McCullough, Finiteness conditions for 3-manifolds with boundary, Amer. J. Math. 109 (1987) 1155-1169 MR919007

[8] W J Floyd, Group completions and limit sets of Kleinian groups, Invent. Math. 57 (1980) 205-218 MR568933

[9] L Greenberg, Commensurable groups of Moebius transformations, from: "Discontinuous groups and Riemann surfaces (Proc. Conf., Univ. Maryland, College Park, Md., 1973)", (L Greenberg, editor), Ann. of Math. Studies 79, Princeton Univ. Press (1974) 227-237 MR0379689

[10] L Greenberg, Finiteness theorems for Fuchsian and Kleinian groups, from: "Discrete groups and automorphic functions (Proc. Conf., Cambridge, 1975)", (W J Harvey, editor), Academic Press, London (1977) 199-257 MR0585138

[11] M Gromov, Hyperbolic groups, from: "Essays in group theory", (S M Gersten, editor), Math. Sci. Res. Inst. Publ. 8, Springer, New York (1987) 75-263 MR919829 
[12] M Gromov, Spaces and questions, Geom. Funct. Anal. (2000) 118-161 MR1826251 GAFA 2000 (Tel Aviv, 1999)

[13] S Helgason, Differential geometry, Lie groups, and symmetric spaces, Graduate Studies in Math. 34, Amer. Math. Soc. (2001) MR1834454 Corrected reprint of the 1978 original

[14] J Hempel, 3-Manifolds, Ann. of Math. Studies 86, Princeton Univ. Press (1976) MR0415619

[15] M Kapovich, On normal subgroups in the fundamental groups of complex surfaces arXiv:math.GT/9808085

[16] C J Leininger, D D Long, A W Reid, Commensurators of non-free finitely generated Kleinian groups arXiv:0908.2272

[17] D D Long, A W Reid, Finding fibre faces in finite covers, Math. Res. Lett. 15 (2008) 521-524 MR2407228

[18] G A Margulis, Discrete subgroups of semisimple Lie groups, Ergebnisse der Math. und ihrer Grenzgebiete (3) 17, Springer, Berlin (1991) MR1090825

[19] D McCullough, Compact submanifolds of 3-manifolds with boundary, Quart. J. Math. Oxford Ser. (2) 37 (1986) 299-307 MR854628

[20] D McCullough, A Miller, G A Swarup, Uniqueness of cores of noncompact 3manifolds, J. London Math. Soc. (2) 32 (1985) 548-556 MR825931

[21] Y Minsky, The classification of Kleinian surface groups. I. Models and bounds, Ann. of Math. (2) 171 (2010) 1-107 MR2630036

[22] M Mj, Cannon-Thurston maps and Kleinian groups arXiv: 1002.0996

[23] M Mj, Cannon-Thurston maps for surface groups arXiv:math.GT/0607509

[24] M Mj, Pattern rigidity and the Hilbert-Smith conjecture arXiv:0906.4243

[25] M Mj, Cannon-Thurston maps and bounded geometry, from: "Teichmüller theory and moduli problem”, (I Biswas, R S Kulkarni, S Mitra, editors), Ramanujan Math. Soc. Lect. Notes Ser. 10, Ramanujan Math. Soc., Mysore (2010) 489-511 MR2667569

[26] G P Scott, Compact submanifolds of 3-manifolds, J. London Math. Soc. (2) 7 (1973) 246-250 MR0326737

School of Mathematical Sciences, RKM Vivekananda University

PO Belur Math, Dt Howrah, WB-711202, India

mahan@rkmvu.ac.in

http://people.rkmvu.ac.in/ mahan/

Proposed: Benson Farb

Received: 16 July 2010

Seconded: Walter Neumann, Jean-Pierre Otal Revised: 1 September 2010 